\title{
La intersubjetividad en las Meditaciones cartesianas y Fenomenología del espíritu
}

\author{
Miguel Herszenbaun \\ CONICET/UBA/GEK/CIF \\ herszen@hotmail.com
}

Recibido 12/06/17

Aprobado 11/07/17

\section{Resumen}

La relación entre subjetividad y alteridad genera interrogantes tanto en lo referente a la esfera constitutiva como en lo que refiere a las implicancias éticas y políticas. En el presente trabajo, nos proponemos confrontar los enfoques desde los que Husserl y Hegel conciben la relación del yo propio con el yo ajeno. Nos concentraremos fundamentalmente en lo que se refiere a la esfera de la constitución del yo y del alter ego. En este sentido, sostendremos que la perspectiva sobre la alteridad propuesta por Hegel en el capítulo IV de la Fenomenología del espíritu supera la posición de Husserl presentada en las Meditaciones cartesianas, en tanto revela una relación de co-constitución entre el propio yo y la autoconciencia extraña que deja abierto un terreno a la experiencia del otro yo no reducible a la propia subjetividad, posibilidad que a nuestro criterio no se encuentra en la mencionada obra de Husserl. A pesar de esta ventaja en el nivel de la constitución, evaluaremos las desventajas ético-políticas de la posición hegeliana. En este sentido, siguiendo los lineamientos indicados por Ricoeur, advertiremos los problemas ético-políticos de la presunta hipóstasis hegeliana de las instituciones políticas o sociales. 
Palabras clave: Subjetividad - Hegel - Husserl - Alteridad - Constitución

\begin{abstract}
The relationship between subjectivity and alterity raises questions referring both to their constitution and their ethical and political consequences. In the following paper, I intend to consider how Husserl and Hegel conceive the relation between the self and the alter ego. In this regard, I will focus mainly in the constitution of the self and the alter ego. I will claim that Hegel's conception of the alterity in chapter IV of the Phenomenology of the Spirit provides a better stand-point from which conceiving the alter ego than Husserl's position presented in Cartesian Meditations. This is due to that Hegel's Phenomenology reveals a relation of coconstitution between the self and the other's self-consciousness that allows a deeper experience of the other that is not reducible to our self-subjectivity. I state that this latter possibility is not presented in Husserl's Cartesian Meditations. Although Hegel's conception of the self has an advantage regarding its constitution, it is necessary to consider its ethical and political disadvantages. In this respect, I will consider Ricoeur's guidelines.
\end{abstract}

Keywords: Subjectivity - Hegel - Husserl - Intersubjectivity - Constitution

\title{
I-Introducción
}

En las Meditaciones cartesianas, Edmund Husserl parece retrotraerse a una inquietud que había preocupado al fundador de la filosofía moderna al que se hace referencia en el título de la mencionada obra. Ya René Descartes había advertido la necesidad de ofrecer un argumento para acreditar la certeza o verosimilitud de nuestro conocimiento del yo extraño. En este sentido, Edmund Husserl dedica los últimos capítulos de sus Meditaciones para volver sobre esta problemática y procurar una fundamentación fenomenológica de la certeza de la experiencia del yo extraño. Por su parte, en El ser y la nada, Jean-Paul Sartre ha considerado necesario revisar la posición husserliana e indicar ciertos límites a dicha posición. En este sentido, Jean-Paul Sartre ha señalado cierto límite a la fenomenología husserliana a la hora de alcanzar sus objetivos en lo referente al tratamiento del yo extraño. A su vez, el autor de El ser y la nada ha propuesto una confrontación entre Husserl y Hegel que también ha sido retomada por Paul Ricoeur. 
En el presente trabajo me propongo volver sobre la confrontación entre Husserl y Hegel en lo referente a la relación entre la propia subjetividad y la subjetividad extraña. Esto será hecho considerando las preocupaciones y señalamientos indicados por Sartre y Ricoeur en lo que se refiere a la discusión entre estos dos autores. Sostendremos que la propuesta hegeliana logra presentar una experiencia del yo extraño más satisfactoria que aquella defendida por Husserl en la medida en que evita caer en una reducción del otro yo a los sentidos originados en el yo propio, y logra conservar un lugar originario de aquel otro toda vez que éste se vuelve constitutivo de la propia autoconciencia. A pesar de esta ventaja en el plano constitutivo, será necesario sopesar si la propuesta hegeliana nos conduce a un problema ético-político en lo referente a la responsabilidad de los individuos particulares en las subjetividades colectivas.

\section{II-Edmund Husserl: la constitución del alter ego y la acusación de solipsismo}

En El ser y la nada Jean-Paul Sartre dedica un capítulo a la comparación de las posiciones de Hegel y Husserl sobre la subjetividad extraña. En aquella obra, la posición del padre de la fenomenología se ve menoscabada por medio de la acusación de solipsismo ${ }^{1}$. En la presente sección nos ocuparemos de presentar la posición husserliana sobre el yo extraño tal como ésta se encuentra en las Meditaciones cartesianas a fin de evaluar qué lugar le queda al alter ego en la propuesta fenomenológica y sopesar la acusación que Sartre esgrime contra él.

En los capítulos IV y V de sus Meditaciones cartesianas Husserl desarrolla su análisis fenomenológico sobre el ego propio y el alter ego. El capítulo IV es dedicado al ego propio. Allí Husserl nos ofrece consideraciones sobre el ego y sobre el método de análisis fenomenológico que resultarán fundamentales para comprender tanto la reducción a la esfera de la propiedad que se llevará a cabo en el capítulo siguiente como para comprender el fundamento de la evidencia que se pretende que tenga la investigación fenomenológica. Husserl afirma que

yo existo para mí mismo y me soy dado constantemente en una evidencia experiencial como yo mismo. [...] Es claro que, por cuanto el concreto ego

\footnotetext{
1 "[Por haber reducido el ser a una serie de significaciones, el único nexo que Husserl ha podido establecer entre mi ser y el del prójimo es el del conocimiento; no puede, pues, evitar mejor que Kant el solipsismo." Sartre, Jean-Paul, El ser y la nada, Barcelona, Losada, 1993, p. 264.
} 
monádico abraza toda la vida real y potencial de la conciencia, el problema de la explicitación fenomenológica de ese ego monádico [...] tiene que abarcar todos los problemas constitutivos en general ${ }^{2}$.

Esto significaría entonces que el análisis del ego -apoyado en la reducción eidética que Husserl desarrolla unas páginas más adelante- nos brinda la evidencia necesaria para el análisis científico y, al mismo tiempo, que la investigación de toda constitución se deberá realizar partiendo del yo.

En el capítulo $\mathrm{V}$ de las Meditaciones es presentada la reducción a la esfera de la propiedad. En dicho procedimiento nos encontramos con una reducción de todo polo objetivo a las vivencias del yo, esto es, a los sentidos que provienen del yo constitutivo de la objetividad. Husserl lo llama "la reducción a mi esfera trascendental propia, o sea, a mi concreto yo-mismo trascendental, abstracción hecha de todo lo que me resulta como extraño en la constitución trascendental" ${ }^{3}$, procedimiento por el que se intenta "ante todo delimitar

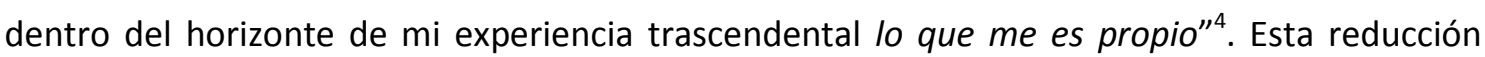
que pretende quedarse únicamente con los sentidos que puedan reconducirse al yo trascendental constituyente de la objetividad se sostiene en la presuposición de un acceso certero a las vivencias propias del yo en cuestión. La certeza del análisis de las vivencias propias conduce a la necesidad de un análisis de la mónada y aquellas trascendencias que pueden reducirse plenamente a los sentidos que emanan únicamente de ella, toda vez que este camino permitirá garantizar la certeza y evidencia deseable para una disciplina científica. Así, la privilegiada certeza de las vivencias propias puede garantizar la cientificidad del análisis de la constitución de las trascendencias, siempre y cuando podamos reducir los polos objetivos al flujo interno de vivencias y a sentidos emanados del ego trascendental ${ }^{5}$, tal como Husserl afirma al caracterizar a la fenomenología como

\footnotetext{
${ }^{2}$ Husserl, Edmund, Meditaciones cartesianas, Madrid, Ediciones Paulinas, 1979, p. 122.

${ }^{3}$ Husserl, Edmund, Meditaciones cartesianas, Op. Cit., pp. 155-156.

${ }^{4}$ Husserl, Edmund, Meditaciones cartesianas, Op. Cit., p. 158.

${ }^{5}$ Las referencias de Husserl sobre la apodicticidad de la investigación propuesta son múltiples y pueden hallarse en diversas secciones de las Meditaciones cartesianas. Mencionamos la siguiente, en la que queda en evidencia la constitución del mundo trascendente y la apodicticidad de su estudio. "[E]l ego [...] tiene un universo de lo que le es propio a sí mismo, el cual puede ser descubierto mediante una
} 
la autoexplicitación de mi ego como sujeto de todo posible conocimiento, llevada a cabo de modo consecuente en la forma de una ciencia egológica sistemática, y esto con respecto al sentido de todo lo que es, que debe poder tener justamente un sentido para mí, el ego ${ }^{6}$.

En consecuencia,

[la] fenomenología universal como autoexplicitación del ego, llevada a cabo en constante evidencia [...] es en primer lugar una explicitación de sí mismo en sentido estricto, que muestra de una manera sistemática cómo el ego se constituye a sí mismo respecto de su propia esencia como siendo en sí y para sí; y luego, en segundo lugar, en una explicitación de sí mismo en sentido lato, que a partir de ahí muestra cómo el ego también constituye en virtud de esa su propia esencia, en él mismo, al otro, lo objetivo y así, en general, todo lo que para él tiene, en cada caso, validez de ser como no-yo en el yo ${ }^{7}$.

Una vez llevada a cabo la reducción a la esfera de la propiedad se buscará "el sentido trascendental de los sujetos extraños" ${ }^{8}$, en otras palabras, se preguntará por el sentido y origen del yo extraño. Practicada la reducción a la esfera de la propiedad emerge inevitablemente la pregunta por el yo extraño, por el origen y sentido de aquel yo que no es el propio. Si el método fenomenológico acude a una reducción en la que se ven expulsados o suspendidos todos los sentidos que no son originarios del yo propio, vale preguntar en qué consiste y en qué se sustenta nuestra experiencia de los otros sujetos (si la hubiere).

explicitación original apodíctica [...] del ego sum apodíctico". Husserl, Edmund, Meditaciones cartesianas, Op. Cit., p. 169.

${ }^{6}$ Husserl, Edmund, Meditaciones cartesianas, Op. Cit., p. 143.

${ }^{7}$ Husserl, Edmund, Meditaciones cartesianas, Op. Cit., p. 143.

${ }^{8}$ Husserl, Edmund, Meditaciones cartesianas, Op. Cit., p. 155. 
Precisamente, el capítulo $V$ busca dar una respuesta a esta pregunta y es aquí donde podremos evaluar la acusación de solipsismo que Sartre dirige contra Husserl.

Podríamos reducir la acusación de Sartre a dos premisas fundamentales. Según Sartre, el tratamiento husserliano del yo extraño resulta solipsista por dos causas interrelacionadas. La primera es la postulación del yo trascendental ${ }^{9}$. La segunda, la definición del ser en función del conocimiento ${ }^{10}$. Partiendo de la distinción del yo empírico y el yo trascendental, siendo que éste es el fundante de aquél, resulta ineludible el problema del solipsismo, toda vez que no podremos tener un acceso originario al yo trascendental ajeno sin caer en una unificación de ambas conciencias. Si a esto le sumamos la determinación del ser como conocimiento, resultaría que el ser de todo sujeto extraño sería reducido al conocimiento de la subjetividad propia.

Ambas premisas llevan al siguiente dilema: o se determina el ser del yo extraño en función del propio conocimiento o bien se pretende que su ser pudiera ser determinado en función de su propio autoconocimiento; pero esta segunda opción, a su vez, implica que su ser resulta incognoscible o, en caso de serme conocido, implique su unificación con el propio flujo de conciencia.

Aun admitiendo que el conocimiento en general mida al ser, el ser del prójimo se mediría en su realidad por el conocimiento que el prójimo tiene de sí mismo, no por el que yo tengo de él. Lo que habría de alcanzar sería el prójimo, no en tanto que tengo conocimiento de él, sino en tanto que él tiene conocimiento de sí mismo, lo que es imposible; supondría, en efecto, la identificación en interioridad entre mí mismo y el otro. ${ }^{11}$

\footnotetext{
9 "[S]i mi ego empírico no es más seguro que el del prójimo, Husserl ha conservado el sujeto trascendental, radicalmente distinto de aquél y harto parecido al sujeto kantiano. Entonces, lo que debería demostrarse no es el paralelismo de los ego empíricos, que nadie pone en duda, sino el de los sujetos trascendentales. [...] Así, el verdadero problema es el de la relación entre los sujetos trascendentales allende la experiencia". Sartre, Jean-Paul, El ser y la nada, Op. Cit., p. 263.

10 "Husserl, por otra parte, se veda la posibilidad de comprender lo que puede significar el ser extramundano del prójimo, ya que define el ser como la simple indicación de una serie infinita de operaciones por efectuar. Imposible hacer más claramente del conocimiento la medida del ser". Sartre, Jean-Paul, El ser y la nada, Op. Cit., p. 263.

${ }^{11}$ Sartre, Jean-Paul, El ser y la nada, Op. Cit., p. 263.
} 
Ahora bien, aun cuando Sartre tal vez pueda acertar al intentar identificar ciertas problemáticas de la fenomenología husserliana, se equivoca en la acusación que imputa a Husserl. En otras palabras, Sartre encuentra problemas posiblemente legítimos en el texto husserliano; sin embargo, imputa a Husserl un cargo inadecuado. Husserl es acusado de solipsismo. Nosotros entendemos que Husserl puede salir indemne de esta acusación. No obstante, creemos que no podrá salir indemne de la acusación de no posibilitar una experiencia verdaderamente originaria del yo extraño.

El cargo de solipsismo implica un privilegio epistemológico de la interioridad del sujeto sobre todo otro conocimiento, ya sea del mundo, de los objetos o de los otros sujetos. Así, el solipsista tendría certeza de su propio yo, pero no certeza de los objetos y sujetos que lo rodean. Estrictamente hablando, entendemos, este no es el caso de Husserl.

El yo analizado por Husserl tiene experiencias de entidades que lo trascienden, cuyo grado de certeza no es distinto al grado de certeza de la experiencia de su propio yo. Esto se debe a que la propuesta fenomenológica radica en describir el proceder de la conciencia, de sus actos noéticos constitutivos del objeto. A través de esta descripción, advertimos que la subjetividad considerada por Husserl tiene una relación inmediata con su objeto, que se le da de manera intuitiva en carne y hueso. La inmediación del objeto ante la conciencia impide sostener que la subjetividad husserliana tenga un acceso de segundo orden, mediatizado, respecto del objeto en comparación con su propia subjetividad.

Ahora bien, el verdadero problema que puede plantearse a la propuesta husserliana se encuentra precisamente en el recurso que permite a Husserl evadir la acusación de solipsismo. Husserl logra fundamentar la experiencia de los objetos trascendentes y de las subjetividades extrañas a través del procedimiento consistente en reducir la experiencia de lo extraño al flujo interno de las vivencias del yo. Dicho en otras palabras, las entidades trascendentes son constituidas con las vivencias de la conciencia constituyente y por medio de la actividad noética, intencional de dicha conciencia. Consecuentemente, toda trascendencia puede ser reconducida a las vivencias inmediatas de la conciencia constituyente. Nuevamente, en otras palabras, todo lo que se presente en la experiencia como extraño a la conciencia puede, en última instancia, ser reconducido, ser concebido como conformado por una vivencia de la conciencia y una actividad constitutiva de la conciencia en cuestión. Por lo tanto, sin importar que lo que se presente a la conciencia sea concebido como algo radicalmente heterogéneo a ella, como algo por completo extraño a ella, en su conocimiento de la entidad extraña la 
conciencia posee cierto grado de certeza que debe equipararse al grado de certeza en el conocimiento de sus propias vivencias. Pues, en última instancia, toda entidad -por extraña que parezca- debe poder ser concebida como el resultado de las vivencias de la conciencia y de sus actos noéticos. En tanto el solipsismo es aquella posición filosófica según la cual se alega tener exclusivamente conocimiento certero de la propia subjetividad, en oposición a una incertidumbre respecto de las entidades extrañas al yo, y en la medida en que Husserl logra garantizar la certeza en el conocimiento de las entidades trascendentes al sujeto, logra el autor de las Meditaciones dar una respuesta satisfactoria a la imputación de solipsismo. Es el carácter intencional de la conciencia, a fin de cuentas, lo que no permite acusar a Husserl de solipsista, si entendemos solipsismo del modo antes indicado.

Dicho en otras palabras, si el solipsismo es la posición filosófica según la cual sólo se encuentra suficientemente fundamentado el conocimiento de sí mismo y todo conocimiento se acota al terreno de la propia subjetividad (mientras todo lo externo a la subjetividad permanece incierto), entonces el propio carácter intencional de la conciencia que presenta en carne y hueso a los objetos intuidos supone, eo ipso, una superación del solipsismo.

Aunque esta reducción al flujo interno de vivencias de la conciencia que fundamenta la certeza de esta experiencia de los objetos y la transferencia aperceptiva que funda la de las otras subjetividades permite responder al cargo de solipsismo, implicará inevitablemente desdibujar la figura del yo extraño para convertirlo en una réplica del ego propio. En otras palabras, Husserl garantiza la experiencia certera de objetos y sujetos extraños, pero no logra garantizar el carácter radicalmente extraño y ajeno del alter ego. Veamos esto de manera detallada.

La $V$ meditación cartesiana tiene, en efecto, la tarea de explicar el modo en que se tiene experiencia del yo ajeno. Una vez hecha la reducción a la esfera de la propiedad, esto es, a la mónada y a su mundo circundante reducible a sentidos que emanen de ella, Husserl debe poder sobrepasar este ámbito de propiedad para acceder a la experiencia de lo extraño. Lo hará acudiendo a una síntesis pasiva de "apareamiento"12 según la cual

dos datos, son dados intuitivamente y destacándose en la unidad de una conciencia [...] ya en pura pasividad, es decir, indiferente a que se lo atienda o

\footnotetext{
${ }^{12}$ El término en alemán es "Paarung".
} 
no, fundamentan fenomenológicamente, en cuanto aparecen como distintos, una unidad de similitud; siempre se constituyen, por tanto, precisamente como $\operatorname{par}^{13}$.

Este proceso también es catalogado como "transgresión intencional" por el que se encuentra "un mutuo y viviente evocarse, y una coincidencia mutua, que intercambia los respectivos sentidos objetivos" ${ }^{14}$. Husserl nos señala que "como resultado de esta coincidencia se efectúa una transferencia de sentido en los elementos apareados, esto es, se lleva a cabo la apercepción de uno de ellos de acuerdo con el sentido del otro" ${ }^{15}$.

La relevancia de este procedimiento radica en que en él se encontrarán las bases de la experiencia del ego extraño. La experiencia del ego extraño será viable en la medida en que el alter ego se presenta en nuestro campo perceptivo, esto es, se presenta como cuerpo físico que deberá adquirir el sentido objetivo cuerpo propio ajeno. Siendo que en la esfera de la propiedad se habían conservado sólo los sentidos que pudieran emanar exclusivamente del ego propio, y en esta esfera aun permanecía el sentido de cuerpo propio fundado en las kinestesias, he aquí que la vía de salida de dicha esfera de la propiedad hacia la experiencia del yo extraño radica en la transferencia del sentido cuerpo propio a un objeto trascendente al yo propio $^{16}$. En otras palabras, el sentido constituido por el propio yo de un cuerpo propio puede ser transferido a un objeto trascendente en virtud de su similitud en su apariencia y comportamiento. Este apareamiento o emparejamiento entre el cuerpo propio nuestro y el objeto extraño que se presenta, atribuyéndole a este último el sentido cuerpo propio, es la

\footnotetext{
${ }^{13}$ Husserl, Edmund, Meditaciones cartesianas, Op. Cit., p. 179.

${ }^{14}$ Husserl, Edmund, Meditaciones cartesianas, Op. Cit., p. 179.

${ }^{15}$ Husserl, Edmund, Meditaciones cartesianas, Op. Cit., p. 179.

16 “Por cuanto en esta naturaleza y este mundo mi cuerpo orgánico es el único cuerpo físico que está constituido y que puede ser constituido originariamente como cuerpo orgánico [...], ese otro cuerpo físico, allí, que sin embargo es aprehendido como cuerpo orgánico, tiene que tener este sentido en virtud de una transferencia aperceptiva a partir de mi propio cuerpo orgánico; [...] sólo una similitud que, dentro de mi esfera primordial, enlace aquel cuerpo físico con mi cuerpo físico puede ofrecer el fundamento de motivación para la aprehensión analogizante del primero como otro cuerpo orgánico". Husserl, Edmund, Meditaciones cartesianas, Op. Cit., pp. 176-177.
} 
constitución del alter ego que fundamenta la experiencia del yo extraño ${ }^{17}$. Según las palabras de Husserl,

\begin{abstract}
si se presenta un cuerpo físico destacado en mi esfera primordial, similar al mío, es decir, con una estructura tal que tenga que entrar en apareamiento fenomenal con el mío, parece sin más estar claro que él, en virtud de la transferencia de sentido, tendrá que tomar de inmediato del mío el sentido 'cuerpo orgánico'18.
\end{abstract}

Husserl es consciente de que esta explicación exige mayores aclaraciones, pues la síntesis pasiva que posibilita el apareamiento no nos pone en un contacto inmediato con las vivencias del otro yo. El apareamiento nos pone frente a un otro yo mentado vacuamente. La intención vacía de este otro yo parte de un cuerpo que sí es intuido en carne y hueso, pero que es incapaz de presentarnos el flujo de vivencias de ese yo extraño. No intuimos la conciencia del otro, sino únicamente su cuerpo. Así, la intuición inmediata del cuerpo extraño, análogo a mi propio cuerpo gobernado por mi propio yo, nos obliga a intencionar vacuamente al yo extraño que habita y gobierna dicho cuerpo propio ajeno y se ve verificada en sus posteriores comportamientos análogos a los míos.

Con esto queda acreditado que Husserl es capaz de superar la acusación de solipsismo $^{19}$. En primer lugar, como hemos mostrado anteriormente, Husserl fundamenta un

\footnotetext{
${ }^{17}$ Según Edith Stein, el pasaje de la subjetividad individual a la experiencia de los yoes extraños se da a través de la empatía sensorial: esto comienza por interpretar los cuerpos vivientes extraños como individuos de mi mismo tipo; luego, con la constitución del nivel sensorial del cuerpo viviente extraño se da, conjuntamente, la constitución de otro yo, como el yo necesario de la sensación. Cfr. Stein, Edith, The Collected Works of Edith Stein, Volume III, "On the problem of empathy", ICS Publications, Washington, D.C., I 989, pp. 59-60.

${ }^{18}$ Husserl, Edmund, Meditaciones cartesianas, Op. Cit., p. 180.

${ }^{19}$ Según Rossi y Muñiz, el procedimiento por medio del cual Husserl, en las Meditaciones cartesianas, intenta fundar la posibilidad de la experiencia del alter ego no logra superar la acusación de solipsismo, en tanto la comunidad intermonádica, esto es, la intersubjetividad, debe tener su fundamento necesario, en última instancia, en la subjetividad trascendental. Esto es, hay una referencia necesaria e insoslayable que remite la intersubjetividad a la subjetividad trascendental constituyente individual. Cfr. Rossi, María José, Muñiz, Marcelo, "Hermenéuticas de la intersubjetividad en Hegel y Husserl”, Revista Eletronica Estudos Hegelianos, año 9, n 16, 2012, pp. 76-78, 83.
} 
conocimiento cierto de las entidades trascendentes al sujeto. En segundo lugar, Husserl es capaz de fundamentar la experiencia de los yoes extraños, aunque con ciertos límites. Pasemos, entonces, a considerar brevemente estos límites.

Creo que la propuesta husserliana no podría hacer lugar a un espacio originariamente extraño del alter ego, entendiéndose por esta falta de originariedad el no poder presentar y fundamentar la experiencia de un alter ego cuyo origen radique en la alteridad propia de esta subjetividad extraña. En otras palabras, la fundamentación de la experiencia de las subjetividades extrañas propuesta por Husserl consiste esencialmente en la fundamentación del experimentar a los otros en tanto estos otros sean en mayor o menor medida analogables a mí. En efecto, Husserl fundamenta la experiencia del otro que lo libera de la acusación de solipsismo. Pero al precio de eliminar toda posible alteridad radical en tales otros, esto es, suprimiendo la posibilidad de la experiencia de un otro radicalmente diferente a mí mismo, no reducible a sentidos originarios propios.

Es decir, Husserl es capaz de fundamentar la experiencia de entidades trascendentes a la propia subjetividad y que éstas sean concebidas y experimentadas como otros yoes, es decir, como subjetividades extrañas. Ahora bien, esta fundamentación tiene como límite y condición el que estos otros sean, al menos en cierto grado, análogos a mí mismo. Con lo cual, Husserl logra fundamentar una experiencia limitada de la subjetividad extraña. Se trata, exclusivamente, de una subjetividad no radicalmente distinta al propio sujeto cognoscente.

Acaso esta acusación pudiera parecer radical y banal al mismo tiempo, pues toda vez que tenemos experiencia y conocimiento de un otro, parecería resultar inevitable que este otro deba ser reconducido a algún sentido que nos resulte propio, sin el cual la experiencia de este otro nos sería en todo punto ajena e incomprensible, incluso al extremo de no ser una experiencia para nosotros en lo absoluto. Pero esta respuesta se sostendría en una indebida comprensión de la acusación aquí esgrimida. Nótese que Husserl lleva a cabo un análisis trascendental en el que se presentan los actos constituyentes del ego, es decir, se presentan

Por nuestra parte, hemos sostenido que Husserl logra superar esta acusación alegando que (1) entendemos solipsismo como aquella posición filosófica según la cual se atribuye certeza exclusivamente al conocimiento de sí en oposición al presunto conocimiento de objetos exteriores $u$ otras subjetividades y (2) que la intencionalidad, esencial a la conciencia, supone un conocimiento certero de lo trascendente, dado como polo objetivo de la conciencia. Lógicamente, Rossi y Muñiz discrepan de nuestras conclusiones, pues entienden que en Husserl hay una preeminencia del conocimiento del propio ego, cuyo carácter apodíctico no puede extrapolarse a los objetos de la conciencia. Cfr. Rossi, María José, Muñiz, Marcelo, "Hermenéuticas de la intersubjetividad en Hegel y Husserl”, Revista Eletronica Estudos Hegelianos, año 9, n 16, 2012, p. 76. 
las actividades de la conciencia a partir de las cuales se constituye y fundamenta el origen de todo polo objetivo y toda trascendencia a la conciencia. La experiencia del alter ego ha sido posibilitada por una síntesis pasiva que constituye al alter ego luego empíricamente experimentado. Con lo cual, lo que está en juego aquí no es la posibilidad de concebir al objeto trascendente experimentado empíricamente en términos comprensibles para nosotros y elaborados por nosotros mismos (lo cual, sería un problema puramente empírico), sino que aquí está en juego la pregunta por la constitución misma de ese alter ego, siendo el resultado de la propuesta husserliana el que dicho yo extraño sólo resulta constituido a través de una traspolación de nosotros mismos - del sentido por medio del cual nos comprendemos a nosotros mismos- a ese objeto trascendente que se vuelve un otro sólo por medio de esta traspolación de sentidos. Esta traspolación implica que el alter ego es constituido como una copia del ego propio. Dicho en otras palabras, si la fundamentación husserliana de la experiencia del yo extraño radica, esencialmente, en que encontramos en nuestra experiencia un objeto físico análogo a nuestro cuerpo, que se comporta como él y que, en consecuencia, sea constituido por la conciencia como un objeto análogo a nuestro propio cuerpo vívido y, por tanto, sobre la base de su intuición intencionamos vacuamente un yo que debe vivir en él y gobernarlo, entonces debe reconocerse que (1) Husserl admite y funda la experiencia del otro, pero (2) sólo de un otro analogable a nosotros (al menos en cierto grado). Es decir, no sólo se nos presenta efectivamente en la experiencia un yo extraño, sino que Husserl logra fundamentar fenomenológicamente dicha presentación, bajo la condición de su semejanza con nosotros mismos: ese otro que se nos presenta, se nos presenta como un yo, porque le atribuimos el significado yo que nos atribuimos a nosotros mismos y es sólo un yo en tanto sea capaz de motivar esta atribución ${ }^{20}$.

Es en este sentido que la acusación que aquí hemos formulado contra Husserl sólo podría ser respondida por medio de una constitución de la alteridad que no reduzca el sentido del otro yo al propio yo, sino que presente una constitución de la alteridad que admita un ámbito propio del otro irreductible a nosotros mismos, una constitución de la alteridad que no

\footnotetext{
${ }^{20}$ Según Theunissen, el tratamiento de la alteridad en Husserl es un medio para alcanzar su verdadera meta, la fundamentación de la objetividad del mundo. Por otra parte, señala Theunissen que el otro, en Husserl, es necesario para mi constitución como un cuerpo en el espacio; requiero del otro para volverme yo mismo un otro, como uno entre otros, y eventualmente como miembro de la comunidad monádica. No obstante, el yo propio es el originariamente constitutivo de todo lo demás, incluso de aquel otro constituyente. Puede decirse que en el origen último de la constitución, según Theunissen, el yo no tiene un par, está solo, y es la fuente constitutiva de todo lo otro que resulte constituyente. Cfr. Theunissen, M., The other. Studies on the social ontology of Husserl, Heidegger, Sartre and Buber, The MIT Press, Cambridge, 1986, pp. 109, 150, 160-161.
} 
esté plenamente en manos de la propia subjetividad. En la sección siguiente, analizaremos si la propuesta hegeliana puede dar una respuesta satisfactoria a nuestra exigencia.

\section{III-Hegel: El surgimiento de la intersubjetividad en la Fenomenología del espíritu}

En esta segunda sección estudiaremos el tratamiento de la intersubjetividad en la Fenomenología del espíritu. Dada la complejidad de la obra mencionada, resulta necesario hacer un breve comentario sobre ciertos aspectos generales del texto en cuestión a fin de lograr comprender con mayor claridad el aporte del capítulo "Autoconciencia" a los objetivos generales de la Fenomenología y en qué consiste la experiencia del otro yo allí presentada.

En su "introducción" la Fenomenología del espíritu presenta de manera suscinta sus intenciones. Entiendo que éstas son lograr la identificación absoluta del ser y el pensar ${ }^{21}$ y que dicha meta puede ser comprendida si atendemos a las críticas que Hegel dirige contra Kant. El giro copernicano kantiano, por medio del cual la conciencia es presentada como constitutiva de la objetividad a través de una síntesis necesaria del múltiple sensible dado, obliga a postular un ámbito de las cosas en sí incognoscible e inaccesible para nosotros. Hegel caracteriza a esta concepción del conocimiento como "temor a la verdad", pues conduciría a separar el conocimiento por un lado y la verdad por el otro ${ }^{22}$. Así, según Hegel, la propuesta kantiana postula una verdad absoluta, pero que paradójicamente nos resulta incognoscible, mientras

\footnotetext{
21 “[L]a meta [...] se halla allí donde el saber no necesita ir más allá de sí, donde se encuentra a sí mismo y el concepto corresponde al objeto y el objeto al concepto". Hegel, G.W.F., Fenomenología del espíritu, México D.F., Fondo de Cultura Económica, 1966, p. 55.

Todas las citas de la sección "Introducción" corresponden a la clásica traducción de Wenceslao Roces editada por Fondo de Cultura Económica. Las restantes citas son tomadas de la traducción de Antonio Gómez Ramos editada por UAM. Se ha optado trabajar con ambas traducciones, pues Roces ha logrado establecer un lenguaje canónico en lo que se refiere a la discusión de la "introducción" a la Fenomenología. Por su parte, el trabajo de Gómez Ramos -en lo que se refiere al capítulo "autoconciencia"- es destacable por su precisión terminológica y aporta claridad a la comprensión del lector hispanoparlante.

22 "Da por supuestas, en efecto, representaciones acerca del conocimiento como un instrumento y un medium, así como también una diferencia entre nosotros mismos y ese conocimiento; pero, sobre todo, presupone el que lo absoluto se halla de un lado y el conocimiento de otro, como algo para sí y que, separado de lo absoluto, es, sin embargo, algo real; presupone, por tanto, que el conocimiento, que, al ser fuera de lo absoluto es también, indudablemente, fuera de la verdad, es sin embargo verdadero, hipótesis con la que lo que se llama temor a errar se da a conocer más bien como temor a la verdad". Hegel, G.W.F., Fenomenología del espíritu, México D.F., Fondo de Cultura Económica, 1966, p. 52.
} 
que por el otro lado el conocimiento que de hecho nos resulta accesible queda degradado a una jerarquía menor, acotado a las meras apariencias.

En contraposición a la concepción kantiana del conocimiento, la tarea de la Fenomenología queda definida entonces como el intento de alcanzar lo absoluto, el cual en tanto absoluto implicará (1) que no haya más verdad o realidad por fuera sí mismo y (2) que incluya o contenga al saber mismo ${ }^{23}$. De esta manera, la meta final de la Fenomenología puede quedar definida como saber absoluto. Dicho saber absoluto no es otra cosa que la meta que logra conciliar el saber con el ser, identificar plenamente ser y saber. Hegel define a la meta de la Fenomenología como la identificación del concepto con el ser. En efecto, esto es otra forma de dar respuesta al intento kantiano (fallido según Hegel) de vincular ser y pensar.

La concepción kantiana de la subjetividad supone que las categorías son únicamente formales y la sensibilidad es necesariamente receptiva. Esto significa que las categorías pueden imponer una unidad a las intuiciones, pero no pueden producir por sí mismas dichas intuiciones. De igual modo, la sensibilidad puede imponer a priori sus formas pero no puede brindar por sí misma el contenido de las intuiciones, necesario para que efectivamente haya conocimiento. En consecuencia, el terreno del conocimiento se ve delimitado por las fronteras de la finitud de la subjetividad. Ahora bien, junto a este acotamiento del conocimiento al terreno delimitado por la receptividad de la intuición sensible, Hegel entiende que la concepción kantiana del conocimiento exige la postulación de un resto incognoscible. Así como la sensibilidad es receptiva respecto de su contenido intuitivo, y así como el conocimiento objetivo es conocimiento de lo aparente, en consecuencia la propia concepción kantiana del conocimiento exige la suposición de una cosa en sí que debe ser postulada como existente, pero debe ser al mismo tiempo concebida como incognoscible. De esta manera, la concepción kantiana del conocimiento obliga a postular una ontología incongruente, contradictoria e incompleta: aquello que se concibe como objeto exige, a su vez, la postulación de otro tipo de entidad que no es objeto de la experiencia, que no puede ser conocido, pero que a la vez debe ser pensado como siendo el fundamento de lo objetivo y el origen de la afección que brinda los datos necesarios para el conocimiento de los objetos.

Contra esta presunta incongruencia, Hegel busca elaborar una estructura conceptual lo suficientemente compleja y completa que impida la constitución de una estructura ontológica que pueda dejar un resto incognoscible, es decir, que permita postular una contracara que se

23 "[S]olamente lo absoluto es verdadero o solamente lo verdadero es absoluto". Hegel, G.W.F., Fenomenología del espíritu, México D.F., Fondo de Cultura Económica, 1966, p. 52. 
escape de nuestras posibilidades de conocer. Siendo ésta la tarea de la Fenomenología, resulta evidente que su estadio final debe ser el saber absoluto entendido como una estructura conceptual que constituya una ontología de la que no pueda restar un ámbito incognoscible. Esta realidad que se identifica con lo absoluto debe ser ella misma a su vez conocimiento, so pena de no ser el verdadero absoluto, alcanzándose entonces una realidad por fuera de la cual no hay nada más, que también es saber y saber de sí misma.

En el marco de estos objetivos perseguidos por la Fenomenología su capítulo IV "autoconciencia" hará un aporte clave. Dicho capítulo comienza con el análisis de una conciencia de objeto que alcanza el estadio de autoconciencia, esto es, conciencia que se conoce a sí misma, para luego pasar al tratamiento del yo ajeno y finalizar con relaciones intersubjetivas más complejas. Este recorrido podría parecer difícilmente compatible con los objetivos generales indicados. Sin embargo, esto no resulta así. El pasaje fenomenológico de una autoconciencia meramente autorreflexiva a un enfrentamiento entre dos autoconciencias es clave en el cumplimiento de los objetivos generales de la Fenomenología. Si la Fenomenología debe alcanzar la identificación absoluta entre ser y pensar, el capítulo IV es el primero en el cual se logra una doble conversión que impulsa al proyecto fenomenológico hacia la meta indicada. La doble conversión a la que hacemos referencia consiste en una conversión del ser en pensar y del pensar en ser. Esta doble conversión se logra únicamente a través de una primera experiencia intersubjetiva en la que en el enfrentamiento de dos autoconciencias cada una de ellas es definida por sí y por la otra como siendo, es decir, como existiendo efectivamente allí en el mundo y como pensamiento. Es decir que la experiencia que la autoconciencia alcanza de las restantes autoconciencias es la conversión de la objetividad en pensamiento, y supone alcanzar una estructura conceptual por medio de la cual se experimenta y se presenta una realidad que no sólo es mera oposición al sujeto, sino que es además sujeto ella misma.

La experiencia de las otras autoconciencias descripta por Hegel dista de ser pacífica. El enfrentamiento de las autoconciencias implica una necesidad mutua y recíproca que, paradójicamente, conduce a un desenlace fatal. Si bien no nos ocuparemos aquí de explayarnos sobre la conocida dialéctica del amo y el esclavo, sí nos ocuparemos de los elementos fundamentales que conducen a ella, pues son estos elementos los que permiten comprender la experiencia del otro descripta por Hegel. La clave de esta experiencia radica en el hecho de que la mera autoconciencia, la mera autorreflexión o autorreferencia del yo sobre 
sí mismo, de la mera tautología 'yo soy yo' se ve plenamente desacreditada por Hegel ${ }^{24}$. Este saber es un saber vacío y sin verdad, esto significa, sin realidad, sin existencia efectiva ${ }^{25}$. La verdad es para Hegel el polo objetivo del saber. Y este saber es una mera reflexión vacía que no tiene, por sí misma, modo de plasmarse en el polo objetivo de la conciencia.

En efecto, la autoconciencia es presentada como un saber que tiene la pretensión de poder superar y suprimir a su objeto. El saber se piensa a sí mismo como más allá del polo objetivo, negando al objeto ${ }^{26}$. Esta negación o eliminación del objeto implica que el saber se refiere a sí mismo como diferente del objeto y atribuyéndole a éste cierta inesencialidad. Sin embargo, así como la continua y perseverante reaparición del objeto negado nos pone sobre aviso del error en el que se encuentra la autoconciencia, Hegel nos alerta que la autoconciencia conserva los estadios anteriores y es tanto una autoconciencia como también una conciencia ${ }^{27}$. Por lo que posee dos objetos. El objeto inmediato, el polo objetivo que trasciende al sujeto, y un segundo objeto, ella misma ${ }^{28}$. Esta división tiene dos consecuencias. La primera de ellas es que la reaparición del objeto negado y catalogado como inesencial a la autoconciencia no es por mera contingencia. La autoconciencia sólo es autoconciencia en la medida en que se refiere a sí misma superando la inmediatez del objeto ${ }^{29}$. La segunda es que

\footnotetext{
24 “[L]a autoconciencia, no es más que la tautología sin movimiento del 'yo soy yo'; en tanto que, a sus ojos, la diferencia no tiene tampoco la figura del ser, ella no es autoconciencia". Hegel, G.W.F., Fenomenología del espíritu, Madrid, UAM, 2010, p. 247.

25 "[La unidad de la autoconciencia consigo misma] es para la autoconciencia un subsistir que, sin embargo, sólo es fenómeno, aparición, o diferencia que no tiene ser en si". Hegel, G.W.F., Fenomenología del espíritu, Madrid, UAM, 2010, p. 247.
}

26 "[E]l objeto de la certeza sensorial y del percibir, el cual, sin embargo, está marcado para ella con el carácter de lo negativo". Hegel, G.W.F., Fenomenología del espíritu, Madrid, UAM, 2010, p. 247.

27 "[E]l saber de otro [...] sus momentos se han preservado" en la nueva figura del saber: "el saber de sí mismo". Hegel, G.W.F., Fenomenología del espíritu, Madrid, UAM, 2010, p. 245. "Con el primer momento, la autoconciencia queda conservada como conciencia [...]; pero, a la vez, sólo en cuanto está referida a su segundo momento, la unidad de la autoconciencia consigo misma". Hegel, G.W.F., Fenomenología del espíritu, Madrid, UAM, 2010, p. 247.

28 "[L]a conciencia tiene, en cuanto autoconciencia, un objeto doble: uno, el inmediato, el objeto de la certeza sensorial [...], y el segundo, a saber, sí misma, que es la esencia verdadera y, de primeras, sólo está presente, por ahora, en la oposición del primer objeto". Hegel, G.W.F., Fenomenología del espíritu, Madrid, UAM, 2010, p. 247.

29 "El deseo y la certeza de sí misma que ha alcanzado en su satisfacción están condicionados por el objeto, pues ella, esa certeza, es cancelando a eso otro; para que tal cancelación tenga lugar, tiene que haber eso otro. La autoconciencia no puede cancelarlo, entonces, por una referencia negativa por parte 
la autoconciencia se ve degradada frente a sus pretensiones originales. Pues, así como el ser se ve definido como distinto del saber y separado de éste, ella, en tanto saber, se ve determinada exclusiva y únicamente como "certeza" y por tanto como excluida del ser y de la verdad ${ }^{30}$.

La mera autoconciencia revela así su vacuidad y su falta de verdad. Pero esta falta de realidad se ve subsanada por la intervención de otros yoes que emergerán a través de un proceso dialéctico que se despliega en el propio polo objetivo. El polo objetivo caracterizado como mera negatividad, como inesencialidad, es constituido de acuerdo con las estructuras conceptuales de la conciencia, esto es, de acuerdo con el saber de la conciencia. Así, la conciencia que se sabe autónoma constituye una ontología acorde a su saber y elabora una objetividad que se le presenta en primer lugar como viviente ${ }^{31}$, en segundo lugar como compuesta de géneros de vida ${ }^{32}$ y en tercer lugar como autónoma ${ }^{33}$.

El complejo proceso dialéctico que Hegel describe en pocas páginas tiene efectos fundamentales. En primer lugar, la mera objetividad es convertida en vida y en vida con géneros (o especies). Hegel presenta un proceso dialéctico en el que la objetividad se ve determinada, por sí misma, como vida y como géneros de vida. Este primer resultado tiene dos consecuencias relevantes. La primera tiene que ver con algo que Hegel nos ha aclarado en la "introducción". La relación entre el saber y el objeto implica que un cambio en el saber conduce a un cambio en el objeto (que no es sino objeto de tal saber); $y$, consecuentemente, una experiencia de un cambio en el objeto implica a su vez un cambio en la estructura conceptual que lo constituye ${ }^{34}$. La determinación del polo objetivo descripta por Hegel

de ella; por eso, antes bien, vuelve a engendrarlo de nuevo, como al deseo". Hegel, G.W.F., Fenomenología del espíritu, Madrid, UAM, 2010, p. 253.

${ }^{30}$ La realización efectiva de la autoconciencia consiste en que su certeza se haga verdad, pero esta verdad sólo puede consistir en una "reflexión duplicada, la duplicación de la autoconciencia" que será explicada más adelante. Hegel, G.W.F., Fenomenología del espíritu, Madrid, UAM, 2010, p. 255.

31 "[E]l objeto del deseo inmediato es algo vivo". Hegel, G.W.F., Fenomenología del espíritu, Madrid, UAM, 2010, p. 247.

32 "Frente a aquella unidad inmediata, o enunciada como un ser, ésta segunda, la universal, es la que tiene dentro de ella todos estos momentos en tanto que asumidos. Ella es el género simple". Hegel, G.W.F., Fenomenología del espíritu, Madrid, UAM, 2010, p. 253.

33 "[La autonconciencia] hace la experiencia de que su objeto es autónomo, se sostiene por sí mismo". Hegel, G.W.F., Fenomenología del espíritu, Madrid, UAM, 2010, p. 253.

34 " $\mathrm{Si}$, en esta comparación, encontramos que los dos términos no se corresponden, parece como si la conciencia se viese obligada a cambiar su saber, para ponerlo en consonancia con el objeto mismo, ya que el saber presente era, esencialmente, un saber del objeto; con el saber, también el objeto pasa a ser 
conduce a la autoconciencia a concebirse a sí misma como vida ${ }^{35}$. La segunda consecuencia es la posibilidad de la concepción de múltiples yoes ${ }^{36}$. Al constituirse los géneros de la vida, por primera vez, podemos pensar el concepto de un yo que no refiera exclusivamente ni se agote plenamente en una referencia a nosotros mismos. El concepto de yo, que claramente debería referir al propio yo y agotarse en él, puede por primera vez permitir pensar al género de los yo $y$, con esto, referir a yoes no propios.

Sin embargo, si esto posibilita pensar o experimentar otros yoes, no lo hace por eso mismo necesario. No obstante, ya encontramos la razón por la que la autoconciencia debe necesariamente tener experiencia de otros yoes. Así como ella se vuelve vida por la necesidad de pensar al objeto como vida y, en consecuencia, adquiere una primera manifestación como realidad efectiva, es decir, existencia efectiva en el $\operatorname{ser}^{37}$, en el polo objetivo, inevitablemente la autoconciencia define y constituye al ser de acuerdo con las categorías que posee y lo constituye en consecuencia como autónomo y sapiente ${ }^{38}$. Si el ser no es definido de esta manera, si el ser no es constituido como sapiente y autónomo, la autoconciencia no podría obtener su realización efectiva en el polo objetivo tal y como ella se concibe. La satisfacción de la pretensión de la autoconciencia de ser saber autónomo se da en su realización efectiva en el ser, con la consecuente necesidad de definir y constituir al ser como pensamiento autónomo. Esta determinación del ser como pensamiento es el surgimiento de las otras autoconciencias.

otro, pues el objeto pertenecía esencialmente a este saber. [...] Así, pues, cuando la conciencia encuentra en su objeto que su saber no corresponde a éste, tampoco el objeto mismo puede sostenerse". Hegel, G.W.F., Fenomenología del espíritu, México D.F., Fondo de Cultura Económica, 1966, p. 58.

35 "Esta otra vida para la que es el género como tal, y que es ella género para sí misma, la autoconciencia [...]”.Hegel, G.W.F., Fenomenología del espíritu, Madrid, UAM, 2010, p. 253.

36 “El yo simple es este género". Hegel, G.W.F., Fenomenología del espíritu, Madrid, UAM, 2010, p. 253. Entendemos que el comprenderse a sí misma de la autoconciencia de acuerdo con el concepto de género posibilitará el emerger efectivo de otras autoconciencias, sin las cuales la propia autoconciencia no puede realizarse como subjetividad en el plano objetivo. La relevancia de esta autcomprensión como género radicará en que posibilita el comprender a un otro como yo sin confundirlo con el propio yo y, en consecuencia, posibilita la multiplicidad de subjetividades.

37 "[U]na certeza tal que ha llegado a ser de modo objetual a los ojos de ella misma". Hegel, G.W.F., Fenomenología del espíritu, Madrid, UAM, 2010, p. 253.

38 “Tanto, pues, como se sostiene en sí misma y es autónoma la conciencia, se sostiene en sí mismo y es autónomo, en sí, su objeto. [...] [L]a autoconciencia [...] hará [...] la experiencia de la autonomía del objeto, descubrirá que éste se sostiene por sí mismo". Hegel, G.W.F., Fenomenología del espíritu, Madrid, UAM, 2010, p. 249. 
Deben existir, deben ser otras autoconciencias allí en el polo objetivo, allí en la realidad efectiva, para que la autoconciencia pueda ella misma ser.

Como es sabido, este encuentro con las otras autoconciencias es menos pacífico de lo que podría esperarse. Dicho encuentro provoca un enfrentamiento entre distintas autoconciencias que se disputan el lugar constituyente y la independencia respecto de la restante autoconciencia. Cada cual se arroga la potestad de constituir la realidad en función de su saber, adjudicándose autonomía respecto del saber ajeno. Dicho enfrentamiento se plasma en la lucha a muerte que pretende dirimir la situación paradójica caracterizada por la necesidad de la autoconciencia de atribuirse a sí misma exclusivamente la autonomía y la aptitud de constituir el polo objetivo y la necesidad de constituir en la realidad efectiva un ser que piense y cuyo reconocimiento también resulta necesario para la autodeterminación como autoconciencia. Esta lucha a muerte es, a su vez, una lucha por la libertad y por la vida, y se manifestará posteriormente en el ámbito del trabajo y la supervivencia ${ }^{39}$. En las secciones siguientes del capítulo IV, Hegel muestra cómo este conflicto dialéctico termina por plasmarse en las figuras del amo y el esclavo, es decir, muestra cómo a través de este conflicto por el reconocimiento, el amo logra el reconocimiento unidireccional por parte del esclavo, se vuelve señor de su vida o muerte y dueño de los frutos de su trabajo; pero, gracias a su trabajo, el esclavo logra volverse dueño de sí mismo y, al ocupar el lugar de mediador entre el amo y la satisfacción de sus necesidades, se vuelve amo del $\mathrm{amo}^{40}$.

Como ya se señaló, aquí no nos interesa desarrollar la dialéctica del amo y el esclavo. Nos interesa estudiar el proceso dialéctico por medio del cual emerge la experiencia de las otras conciencias. Este procedimiento tiene múltiples niveles. Por un lado, surge en el marco

\footnotetext{
${ }^{39}$ En términos de Kojève, "el hombre, por ser libre y autónomo, reconoce que los otros son igualmente libres y autónomos. E inversamente sólo es libre y autónomo si es libremente reconocido como tal por los otros. El movimiento dialéctico es doble; es una interacción. Es el hombre social, histórico", Kojève, A, La dialéctica del amo y del esclavo en Hegel, Fausto Ediciones, Buenos Aires, 1999, p. 52. En consecuencia, debe entenderse que en el conflicto entre autoconciencias se pone en juego la vida en su sentido más fundamental y brutal, pero también se pone en juego el reconocimiento en la esfera social, jurídica, política, etc. También cabría entender que Kojève considera la posición de Hegel como sosteniendo una intersubjetividad intrínseca a la propia subjetividad, que necesita del otro para volverse tal en sentido pleno.

${ }^{40}$ Dice Kojève "El amo sigue siendo esclavo de la naturaleza por su Begierde. El esclavo: su trabajo no es destrucción del objeto (como la Begierde); el trabajo lo forma, lo modifica. Transforma la Natur en Welt (el universo natural en un mundo histórico). Por eso de hecho se libera de la naturaleza. Por el trabajo deviene también consciente de su libertad", Kojève, A, La dialéctica del amo y del esclavo en Hegel, Fausto Ediciones, Buenos Aires, 1999, p. 57.
} 
del emerger de la vida, con lo cual se pone en juego en él la vida misma de la conciencia. Pero tiene también un carácter epistemológico y ontológico. Se fundamenta en una modificación en el conocimiento que la conciencia tiene de sí. Pero conduce a una consecuencia ontológica: una modificación de la constitución del ser objetivo en función de dicho nuevo saber.

Ahora bien, esta modificación del polo objetivo (de acuerdo con la cual el objeto se presenta como autoconciente) supone un aporte clave a las metas generales de la Fenomenología del espíritu, pues consiste en la primera conversión del ser en el pensamiento y del pensamiento en el ser, abriendo el camino hacia la absoluta identificación de ser y pensar. La experiencia descripta en el capítulo "autoconciencia" es a nuestro criterio la experiencia de un yo extraño que se presenta trenzado y enraizado con la propia constitución como autoconciencia. Es decir, el yo extraño está ontológicamente vinculado a la conciencia propia, de modo tal que ambas se requieren para ser lo que son.

Con esto queremos decir que la propuesta de Hegel es profundamente distinta de la propuesta husserliana. El yo ajeno no es el resultado de una autoconstitución extrapolada a fin de constituir al yo extraño. En la Fenomenología de Hegel, la constitución de la propia subjetividad no puede hacerse sin el emerger de los yoes extraños. La mera autorreflexión o autorreferencia interna al yo propio no es suficiente para la constitución plena y verdadera de la subjetividad propia. El emerger del otro es ineludible. La propuesta hegeliana exige la experiencia de un otro para la conformación de la identidad propia, y no nos atreveríamos a afirmar que ese otro es una mera réplica del yo propio, pues, muy por el contrario, el yo propio sólo se encuentra plenamente constituido luego de la intervención del otro. En consecuencia, podríamos hablar de un proceso co-constituyente del yo y del otro, esto es, un proceso que no parte de un yo (el propio) para llegar al otro (el ajeno), sino un proceso de mutua influencia en el que ambos yoes emergen por la recíproca interacción.

\section{IV-Ricoeur: respuesta de la fenomenología husserliana a la fenomenología hegeliana}

En su trabajo "Hegel y Husserl sobre la intersubjetividad" Paul Ricoeur se dedica a abordar las posiciones de estos dos autores respecto del tratamiento de la subjetividad ajena. Así como hemos hecho una breve referencia a la interpretación sartreana del texto de Husserl, nos parece necesario dedicar algunas palabras a la discusión que plantea Ricoeur entre las posiciones mencionadas. 
Resulta interesante destacar que en su análisis del tratamiento hegeliano de la intersubjetividad Ricoeur opta por no hacer referencia al capítulo IV de la Fenomenología del espíritu, sino ocuparse principalmente del capítulo V. Esta decisión no resulta menor pues determinará la perspectiva del análisis ricoeuriano sobre la posición de Hegel. En términos generales, podemos afirmar que la oposición que Ricoeur presenta entre Husserl y Hegel se encuentra primordialmente en el tratamiento de las entidades colectivas. Mientras la posición hegeliana es caracterizada por Ricoeur como la hipóstasis de entidades colectivas, esto es, una sustancialización de grupos, elevándolos a la categoría de entidades cuya existencia no se acota a la mera acumulación e interrelación de individuos particulares, la posición de Husserl es presentada como de una descomposición de estas presuntas entidades colectivas en sus componentes particulares y explicándolas en términos de interacción de individuos ${ }^{41}$. En otras palabras, aquello que en Hegel es hipostasiado y presentado como teniendo una entidad propia y autónoma respecto de los particulares, en Husserl es explicado a través de la interacción de mónadas en una comunidad intermonádica.

A nuestro criterio, la decisión de tratar el capítulo $V$ de la Fenomenología y pasar por alto el capítulo IV tiene mucho peso en la interpretación propuesta por Ricoeur. Pues sólo puede admitirse la interpretación propuesta por Ricoeur -esto es: Hegel hispotasia grupos de individuos para convertirlos en entidades colectivas autónomas de sus miembros- si se comete el error de obviar el capítulo IV de la Fenomenología del espíritu. En él, como hemos visto, se parte de una autoconciencia individual, con el objetivo de demostrar que la

\footnotetext{
${ }^{41}$ Rossi y Muñiz entienden que así como en la Ciencia de la lógica la otredad es co-originaria del algo, en la Fenomenología del espiritu la intersubjetividad es co-originaria de la subjetividad. En la Fenomenología, el despliegue de figuras no está fundado en el sujeto singular, sino que se trata de un movimiento de despliegue y exposición de la cosa misma, de la experiencia misma. Este despliegue, que no es otra cosa que el brotar de la vida, lleva al surgimiento del sujeto, acompañado insoslayablemente de la intersubjetividad. La diferencia en lo que respecta a la concepción de la experiencia entre Hegel y Husserl es lo que conduce a estos autores a entender que la interpretación de Ricoeur sobre la articulación de la obra de Husserl y Hegel es parcial. Ricoeur intenta leer a Hegel desde la fenomenología de Husserl. Rossi y Muñiz, por el contrario, entienden que la fenomenología husserliana bien podría ser tomada como un momento del despliegue del espíritu en la Fenomenología hegeliana, esto es, como el momento "conciencia". Cfr. Rossi, María José, Muñiz, Marcelo, "Hermenéuticas de la intersubjetividad en Hegel y Husserl", Revista Eletronica Estudos Hegelianos, año 9, n 16, 2012, pp. pp. $68-69,75,84,91$.
}

Por otra parte, cabe señalar que la idea sugerida por Rossi y Muñiz según la cual habría una articulación entre Lógica y Fenomenología tal que las formas lógicas encontrarían un cierto correlato o manifestación en la Fenomenología también ha sido defendida por Fulda. En este sentido, puede verse Fulda, H., "Zur Logik der Phänomenologie von 1807", Fulda, H, Henrich, D., Materialien zu Hegels Phänomenologie des Geistes, Frankfurt am Main, 1973. 
postulación misma de una única autoconciencia conduce a la necesaria constitución de una pluralidad de autoconciencias. En otras palabras, se muestra el enraizamiento ontológico necesario entre múltiples subjetividades, haciendo notorio (1) que la subjetividad individual está necesariamente inserta y vinculada con la intersubjetividad, pero que (2) la intersubjetividad es el producto de la interacción de múltiples sujetos individuales y no una instancia colectiva con entidad propia.

Una vez ganado el suelo mínimo de intersubjetividad que ofrece el capítulo IV, se da paso al tratamiento de relaciones sociales más elaboradas, siendo el capítulo mencionado un elemento esencial en la fundamentación del análisis de dichas instancias sociales. Es precisamente el desarrollo dialéctico que lleva al surgimiento de una autoconciencia extraña, lo que posteriormente conduce al surgimiento de las instancias sociales y comunitarias, con lo cual, desde la perspectiva de Hegel, estaría justificado el pasaje a lo que Ricoeur llama entidades colectivas (i.e. el Estado, el pueblo, la familia, gremios, estamentos, etc.).

De esta forma, Ricoeur cae en el error de creer que las entidades colectivas presentadas por Hegel son una mera hipóstasis infundada, porque no se ha detenido en comprender el proceso dialéctico que conduce de la conciencia hacia la autoconciencia, de la autoconciencia hacia la experiencia de otras autoconciencias, y así a su vez a la interacción de múltiples autoconciencias. En otras palabras, Ricoeur no ha podido comprender el movimiento del espíritu, ya presente en el capítulo IV e impulsando el progreso de la Fenomenología hacia las figuras subsiguientes.

En franca oposición a Hegel, Ricoeur presenta la posición de Husserl en la que la entidad colectiva es comprendida como la interrelación de mónadas autónomas, esto es, una comunidad intermonádica, concluyendo Ricoeur que

la ventaja decisiva de Husserl sobre Hegel consiste en su rechazo sin compromiso de hispotasiar las entidades colectivas y en su voluntad tenaz de reducirlas siempre a una red de interacciones. Este rechazo y esta voluntad tiene una significación crítica considerable. La sustitución del espíritu objetivo hegeliano por la intersubjetividad preserva, en mi opinión, los criterios mínimos de la acción humana, a saber, que se la pueda identificar mediante proyectos, 
intenciones, motivos de agentes capaces de imputarse a ellos mismos su acción. ${ }^{42}$

El trabajo de análisis que Ricoeur ofrece sobre estos dos autores se ve motivado por una preocupación ética de mayor importancia. Según Ricoeur, la posición hegeliana conduciría a comprender las instituciones sociales como entidades colectivas independientes de la actividad de los miembros y, en consecuencia, haciendo irresponsables a los particulares que participan de ellas de las consecuencias que produzcan dichas instituciones. De igual modo, el curso de la historia, el quehacer de las sociedades parecería, desde esta perspectiva, escaparse por completo de las manos del ser humano, del individuo particular, pues si las instituciones operaran con independencia de sus miembros, no se vería cómo los seres humanos y las sociedades podrían controlar sus propios destinos. Ante los estragos de las guerras, los genocidios y otras calamidades que el ser humano pudo concretar es sumamente relevante la preocupación que parece querer manifestar Ricoeur.

Teniendo en mente esta preocupación, Ricoeur cree encontrar una respuesta más satisfactoria en términos éticos en el tratamiento husserliano de la intersubjetividad que en el de Hegel. Pues, según Ricoeur, al ser capaz de interpretar las instituciones sociales en términos de interrelación de individuos, Husserl puede imputar responsabilidades a individuos concretos y reales, de modo tal que no sólo el ser humano responde por los proyectos encausados por las instituciones de las que participa, sino que además el ser humano a través de dicha responsabilidad se hace dueño de su destino.

Lo cierto es que la lectura que Ricoeur hace de Hegel no es acertada por varios motivos. En primer lugar, debemos remitirnos a un punto antes mencionado: el surgimiento de la intersubjetividad en Hegel no supone la supresión o eliminación de la subjetividad individual. Por el contrario, las instancias intersubjetivas comunitarias tienen su origen en el reconocimiento recíproco presente en el capítulo IV de la Fenomenología ${ }^{43}$. A esto se agrega

\footnotetext{
${ }^{42}$ Ricoeur, Paul, "Hegel y Husserl sobre la intersubjetividad” en Del texto a la acción, México, FCE, 2000, pp. 277-278.

${ }^{43}$ En este sentido se pronuncia Valls Plana al afirmar que "el reconocimiento [...] es lo que traba las autoconciencias entre sí y constituye la unidad del espíritu. La perfecta libertad e independencia de los sujetos en el seno del espíritu se realizará en el reconocimiento mutuo", Valls Plana, R., Del yo al nosotros. Lectura de la Fenomenología del espíritu de Hegel, Editorial Laia, Barcelona, 1979, p. 115. Es decir, las figuras más desarrolladas que realizan al espíritu no suponen la supresión del individuo, sino el medio en que se realiza su libertad.
} 
que las sucesivas figuras que se presentan en el desarrollo de la Fenomenología del espíritu se dirigen hacia un nuevo reconocimiento del individuo. Esto puede verse por ejemplo en la caída de la comunidad griega y el surgimiento del derecho romano o el surgimiento del cristianismo. En ambos casos, se trata de nuevas formas de reconocer al individuo como tal, ya sea como persona jurídica con derechos subjetivos, ya sea como un alma particular con una relación inmediata con lo divino ${ }^{44}$. Esto es, el desarrollo de la Fenomenología no se dirige a una supresión de las individualidades, sino a un reconocimiento de ellas al interior de comunidades, en figuras cada vez más complejas. En segundo lugar, Ricoeur pasa por alto el momento de la moralidad en la Filosofía del derecho de Hegel. Allí, luego de haber presentado las relaciones jurídico-formales del Derecho abstracto y antes de haber ingresado en la esfera ético-política, Hegel presenta el momento de la moralidad. La moralidad se caracteriza por la interiorización del deber jurídico como un deber que obliga al individuo de manera personal. Como es de esperar, la moralidad no es suficiente para la conformación del orden social; pero sí supone que en las instancias intersubjetivas que conforman las complejas relaciones económicas, sociales y políticas que se presentan en las sociedades modernas, el sujeto es moralmente responsable de su accionar, aun siendo parte de un engranaje más complejo. En tercer lugar, Ricoeur parece caer en un clásico lugar común anti-hegeliano, según el cual se acusa a Hegel, como filósofo de la historia, de concebir al desarrollo histórico de la humanidad como un proceso independiente de la actividad concreta de los individuos que conforman los pueblos y las comunidades. Lo cierto es que esta lectura de la filosofía de la historia de Hegel desconoce los señalamientos anteriores sobre la Fenomenología del espíritu. Además, pasa por alto que el proceso dialéctico por medio del cual se avanza en la Fenomenología no es un proceso mecánico, causal, que avanza de manera inexorable, más allá de la voluntad de los sujetos implicados. El proceso dialéctico conduce a contradicciones, demuestra la insuficiencia de las sucesivas figuras, etc., pero no puede obligar a una comunidad a llevar a un cambio legislativo, cultural, productivo, en contra de la voluntad de los individuos. En otras palabras, las contradicciones que llevan a la caída de una figura histórica no son independientes de los sujetos actuantes que, por un lado, llegan a esa contradicción y, por el otro, provocan su superación.

\footnotetext{
${ }^{44}$ Sobre el surgimiento del derecho liberal y el reconocimiento del individuo puede consultarse el trabajo Herszenbaun, M., "Derecho abstracto y estado jurídico en el pensamiento político hegeliano", Universitas. Revista de Filosofía, Derecho y Política, n²1, 2015, pp. 21-34.
} 


\section{V-Conclusiones}

Como hemos podido apreciar, Husserl es capaz de evadir la acusación de solipsismo formulada por Sartre. En este sentido, la intencionalidad y la transferencia aperceptiva permiten fundamentar el conocimiento de objetos exteriores y subjetividades extrañas. No obstante, el mismo procedimiento que exonera a Husserl del cargo de solipsismo, conduce a plantear otra inquietud: en qué medida el otro experimentado de acuerdo con la fenomenología husserliana es radicalmente un otro. Este otro debía ser en cierto grado análogo a mí, lo que impedía fundamentar la experiencia de una subjetividad radicalmente distinta a mí. Ese otro, incapaz de tener un fundamento en sí mismo, debe semejarse a mí.

Hegel da, en este sentido, una respuesta más satisfactoria. En tanto la Fenomenología demuestra que el otro es co-constitutivo de la experiencia, que la subjetividad extraña es cooriginaria junto con la propia autoconciencia, resulta que el otro no emerge como una copia de mí mismo, sino que las autoconciencias propia y ajena emergen conjuntamente y se requieren recíprocamente para este mutuo emerger. De esta manera, advertimos que la concepción hegeliana de la intersubjetividad abre una puerta a la fundamentación de una alteridad radical, esto es, de una subjetividad radicalmente otra que no sea, sin más, una copia de mí mismo, que tenga un fundamento en sí misma, no menos fundante y originario que el fundamento que se encuentra en mí. En otras palabras, la fundamentación y origen de las autoconciencias no se reduce a mí, sino que se encuentra entrelazado entre ambas autoconciencias.

Sin embargo, frente a la propuesta hegeliana, se levanta una objeción. Ricoeur ve en la intersubjetividad hegeliana una hipóstasis de entidades colectivas, la conversión de lo que no sería más que una red de interacción de sujetos particulares en una sustancia autónoma a dichos sujetos y con entidad propia, con graves consecuencias ético-políticas: la descarga de la responsabilidad ética, jurídica y política de los particulares sobre la entidad colectiva.

Frente a esta acusación, hemos podido señalar que la lectura que Ricoeur hace de Hegel resulta parcial, toda vez que obvia la importancia del capítulo IV de la Fenomenología, "Autoconciencia". Dicho capítulo resulta clave para comprender el surgimiento de la intersubjetividad, pero más aún para comprender el lugar del sujeto particular en la Fenomenología, su pervivencia a lo largo de la obra, en los restantes capítulos, su volver a emerger en las figuras cada vez más complejas del espíritu. Dicho en pocas palabras, el reconocimiento de la individualidad no se desvanece en el espíritu, sino que vuelve a emerger una y otra vez. Lo que supone que ni el respeto a la individualidad, ni la responsabilidad individual pueden desaparecer en el tratamiento hegeliano de la intersubjetividad. 


\section{Bibliografía}

- FULDA, H., "Zur Logik der Phänomenologie von 1807”, Fulda, H, Henrich, D., Materialien zu Hegels Phänomenologie des Geistes, Frankfurt am Main, 1973

- HEGEL, G.W.F., Fenomenología del espíritu, Madrid, UAM, 2010,

- $\quad$--------------., Fenomenología del espíritu, México D.F., Fondo de Cultura Económica, 1966

- HERZENBAUN, M., "Derecho abstracto y estado jurídico en el pensamiento político hegeliano", Universitas. Revista de Filosofía, Derecho y Política, n²1, 2015

- HUSSERL, Edmund, Meditaciones cartesianas, Madrid, Ediciones Paulinas, 1979

- $\quad$ KOJÈVE, A, La dialéctica del amo y del esclavo en Hegel, Fausto Ediciones, Buenos Aires, 1999

- ROSSI, María José, Muñiz, Marcelo, "Hermenéuticas de la intersubjetividad en Hegel y Husserl”, Revista Eletronica Estudos Hegelianos, año 9, n 16, 2012

- $\quad$ SARTRE, Jean-Paul, El ser y la nada, Barcelona, Losada, 1993

- STEIN, Edith, The Collected Works of Edith Stein, Volume III, "On the problem of empathy", ICS Publications, Washington, D.C., I 989,

- THEUNISSEN, M., The other. Studies on the social ontology of Husserl, Heidegger, Sartre and Buber, The MIT Press, Cambridge, 1986

- VALLS PLANA, R., Del yo al nosotros. Lectura de la Fenomenología del espíritu de Hegel, Editorial Laia, Barcelona, 1979, 